\title{
COMPLEMENTATION FOR RIGHT IDEALS IN GENERALIZED HILBERT ALGEBRAS
}

BY

\section{JOHN PHILLIPS}

ABSTRACT. Let $\mathfrak{a}$ be a generalized Hilbert algebra and let $\mathcal{I}$ be a closed right ideal of $\mathfrak{A}$. Let $\mathcal{I}^{\perp}$ denote the pre-Hilbert space orthogonal complement of $\mathfrak{J}$ in $\mathscr{\vartheta}$. The problem investigated in this paper is: for which algebras $\mathfrak{Q}$ is it true

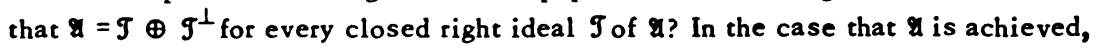
a slightly stronger property is characterized and these characterizations are then used to investigate some interesting examples.

Introduction. It is known that for full Hilbert algebras, the complementation property holds for both closed left and right ideals. This fact is due to Yood [7]. For achieved generalized Hilbert algebras the problem is more subtle although not difficult. A number of characterizations of a strong complementation property are given below. Perhaps the most useful of these is Theorem 1.11. Several cases are analyzed using this theorem. I would like to thank T. W. Palmer for suggesting this problem to me.

Definitions and notation. The definitions and notation of M. Takesaki's printed notes [6] will usually be used without reference. In particular, if $\mathcal{Q}$ is a generalized Hilbert algebra, its involution will be noted by \# and for $\xi \in \mathfrak{A}, \pi(\xi)$ will denote the unique continuous extension to the completion of $\mathscr{U}$ of the operator "left multiplication by $\xi$ ". If $b$ is the conjugate linear adjoint of \# and if $\gamma$ is in the domain of $b$ then one can define an operator on $\mathcal{U}$ by: $\pi^{\prime}(\mathcal{\eta}) \xi=\pi(\xi) \Re$ for all $\xi \in \mathfrak{N}$. If $\pi^{\prime}(\mathcal{l})$ is bounded on $\mathfrak{U}$ then the unique bounded extension of $\pi^{\prime}(\mathfrak{l})$ to the completion of $\mathcal{U}$ is denoted by $\pi^{\prime}(\eta)$ also. The set of all $\eta$ in the domain of b such that $\pi^{\prime}(\eta)$ is bounded will be denoted by $\mathfrak{U}^{\prime}$. By Lemma 3.3 of [6], $\mathfrak{Y}^{\prime}$ is an algebra with involution $b$ such that $\left(\mathscr{R}^{\prime}\right)^{2}$ is dense in the domain of $b$. In particular, $\mathfrak{U}^{\prime}$ is dense in the completion, $\mathcal{H}(\mathscr{U})$ of $\mathscr{U}$. If $\xi$ is any element of $\mathcal{H}(\mathscr{U})$ one can define an operator $\pi(\xi)$ with domain $\mathfrak{U}^{\prime}$ by: $\pi(\xi) \eta=\pi^{\prime}(\eta) \xi$ for all $\eta \in \mathfrak{U}^{\prime}$. If $\pi(\xi)$ is bounded on $\mathfrak{U}^{\prime}$ then the unique bounded extension of $\pi(\xi)$ to $\mathcal{H}(\mathscr{U})$ is denoted by $\pi(\xi)$ also. The set of all elements $\xi$ of $\mathcal{H}(\mathscr{U})$ such that $\pi(\xi)$ is bounded will be denoted by $\mathcal{H}_{l}$.

Received by the editors January 4, 1973. $46 \mathrm{H} 10$.

AMS (MOS) subject classifications (1970). Primary 46K15, 46L10; Secondary 46K05, 
If $\mathscr{X}$ is a generalized Hilbert algebra, a subset $\mathcal{K}$ of $\mathcal{H}(\mathscr{U})$ is said to be right invariant; left invariant; or two-sided invariant respectively if $\pi^{\prime}\left(\mathfrak{X}^{\prime}\right) \mathcal{K} \subseteq \mathcal{K}$; $\pi(\mathfrak{U}) \mathcal{K} \subseteq \mathcal{K}$; or both $\pi^{\prime}\left(\mathfrak{U}^{\prime}\right) \mathcal{K} \subseteq \mathcal{K}$ and $\pi(\mathfrak{Q}) \mathcal{K} \subseteq \mathcal{K}$. If $\mathcal{H}$ is any Hilbert space and $\mathcal{K}$ is any subset of $\mathcal{H}$ then $\mathcal{K}^{\Phi}$ is defined by $\bar{K}^{\oplus}=\{\xi \in \mathcal{H} \mid(\xi \mid \mathcal{N})=0$ for all $\pi \in \mathcal{K}\}$. If $\mathscr{U}$ is any generalized Hilbert algebra and $\mathcal{H}$ is the completion of $\mathcal{U}$, then for any subset $\mathfrak{T}$ of $\mathscr{U}, \mathfrak{I}^{\perp}$ is defined by $\mathfrak{T}^{\perp}=\mathfrak{T}^{\Phi} \cap$ Ul. Moreover, $\overline{\mathfrak{T}}^{\mathcal{H}}$ is defined to be the closure of $\mathfrak{T}$ in $\mathcal{H}$ and $\overline{\mathfrak{T}}=\overline{\mathfrak{T}}^{\mathcal{H}} \cap \mathfrak{U}$ is the relative closure of $\mathfrak{T}$ in $\mathfrak{A}$.

1. The complementation property.

1.1 Lemma. Let थ be a generalized Hilbert algebra. If $\mathcal{T}$ is a right (left; two-sided) ideal of $\mathfrak{A}$, then $\mathfrak{I}^{\perp}$ is a closed right (left; two-sided) ideal of $\mathscr{N}$. More. over, $\overline{\mathcal{J}}^{\mathcal{H}}$ and $\mathcal{J}^{\oplus}$ are closed right (left; two-sided) invariant subspaces of $\mathcal{H}(\mathfrak{Q})$.

Proof. Since $\pi$ is a $*$-representation of $\mathfrak{U}$ as bounded operators on $\mathfrak{H}$, the proof for left ideals is easy. Thus, it suffices to give the proof for right ideals.

Let $\mathfrak{J}$ be a right ideal of $\mathfrak{U}$. It is first shown that $\overline{\mathfrak{J}}^{\mathfrak{l}}$ is a right invariant subspace of $\mathcal{H}$. Let $\Re \in \mathfrak{U}^{\prime}$ and let $\xi \in \mathfrak{T}^{\mathcal{H}}$. Let $\left\{\xi_{n}\right\} \subseteq \mathcal{T}$ and $\left\{\Re_{n}\right\} \subseteq \mathfrak{U}$ be sequences such that $\lim _{n} \xi_{n}=\xi$ and $\lim _{n} \pi_{n}=\pi$. Then,

$$
\pi^{\prime}(\pi) \xi=\lim _{n} \pi^{\prime}(\Re) \xi_{n}=\lim _{n} \pi\left(\xi_{n}\right) \pi=\lim _{n} \lim _{k} \pi\left(\xi_{n}\right) r_{k} .
$$

But, $\pi\left(\xi_{n}\right) \Re_{k}=\xi_{n} \Re_{k} \in \mathcal{T}$ so that $\pi^{\prime}(\Re) \xi \in \widetilde{T}^{\mathcal{H}}$. Therefore, $\mathscr{T}^{\mathcal{H}}$ is right invariant and since $\pi^{\prime}$ is an anti-*-representation of $\mathfrak{U}^{\prime}$ on $\mathcal{H}$ we have that $\mathcal{J}^{\mathscr{\Theta}}$ is also right invariant.

To see that $\mathcal{T}^{\perp}$ is a right ideal of $\mathfrak{X}$, let $\zeta \in \mathcal{T}^{\perp}$ and let $\xi \in \mathfrak{P}$. Let $\left\{\xi_{n}\right\} \subseteq$ $\mathcal{U}^{\prime}$ be a sequence such that $\lim _{n} \xi_{n}=\xi$. Then, $\zeta \xi \in \mathscr{U}$ and $\zeta \xi=\lim _{n} \pi(\zeta) \xi_{n}=$ $\lim _{n} \pi^{\prime}\left(\xi_{n}\right) \zeta$. But, $\pi^{\prime}\left(\xi_{n}\right) \zeta \in \mathfrak{T}^{\oplus}{ }_{\text {so that }}^{n} \zeta \xi \in \mathfrak{T}^{\Phi} \cap \mathfrak{U}=\mathfrak{T}^{\perp}$.

1.2 Lemma. Let $\mathfrak{Q}$ be a generalized Hilbert algebra sucb tbiat $\pi(\mathfrak{l})$ is a left ideal in $L(\mathscr{Q})$. Let $E$ be a closed right invariant subspace of $\mathcal{H}(\mathfrak{Q})$. Then $E \cap$ OV is dense in $E$ and is moreover a closed right ideal of 2 .

Proof. Let $P$ be the orthogonal projection on $E$. Since $P$ commutes with $\pi^{\prime}\left(\mathscr{U}^{\prime}\right), P$ is in $L(\mathscr{Z})$. Hence $\pi(P(\mathscr{U}))=P \pi(\mathscr{U}) \subseteq \pi(\mathscr{U})$ or $P(\mathscr{U}) \subseteq \mathscr{X}$. Thus, $P(\mathfrak{U}) \subseteq$ U⿱ $\cap E$ is dense in $E$ since $\mathscr{U}$ is dense in $\mathcal{H}$. It is fairly clear that $\mathscr{U} \cap E$ is a closed right ideal of 2 .

1.3 Definition. Let $\mathfrak{A}$ be a generalized Hilbert algebra. Then, $\mathfrak{U}$ is said to be strongly orthocomplemented if for every closed right ideal $\mathfrak{T}$ of $\mathscr{N}$, it is true that $\mathfrak{X}=\mathfrak{T} \oplus \mathfrak{T}^{\perp}$, and for every nonzero projection $P$ in $L(\mathfrak{U}), P(\mathfrak{U}) \cap \mathfrak{U} \neq\{0\}$.

1.4 Example. An achieved generalized Hilbert algebra $\mathfrak{U}$ and a nonzero projection $P$ in $L(\mathscr{U})$ such that $P(\mathscr{U}) \cap \mathscr{U}=\{0\}$.

Let $\mathcal{H}$ be a separable Hilbert space with orthonormal basis $\left\{x_{n}\right\}_{n=1}^{\infty}$. 
Let $\mathscr{S}(\mathcal{M})$ denote the von Neumann algebra of all bounded linear operators on $\mathcal{H}$ and define a function $\phi$ on the positive elements $T$ of $\mathscr{B}(\mathcal{H})$, via $\phi(T)=\sum_{n=1}^{\infty} n\left(T x_{n} \mid x_{n}\right)$. Then $\phi$ satisfies the hypotheses of Theorem 2.13 of [2] and so if $\mathscr{U}=\{T \in \mathscr{B}(\mathcal{H}) \mid$ $\left.\phi\left(T^{*} T\right)+\phi\left(T T^{*}\right)<\infty\right\}$ then $\mathscr{U}$ is an achieved generalized Hilbert algebra with linear product $(T \mid S)=\phi\left(S^{*} T\right)$ ( $\phi$ can be uniquely extended to $\left.\mathscr{U}\right)$ and $L(\mathscr{U})$ is naturally isomorphic to $\mathfrak{B}(\mathcal{H})$. Let $P$ be the projection on the vector $\Sigma_{n=1}^{\infty} n^{-1} x_{n}$. Then, $P \in \mathscr{B}(\mathcal{H})$ but, $P \notin \mathscr{U}$ since $\phi(P)=\infty$. Now, $P(\mathscr{U}) \cap \mathscr{U}$ is a right ideal of $\mathscr{U}$ and so by Theorem 1.14 of this paper there is a nonzero projection $Q \in P(\mathfrak{U}) \cap \mathscr{U}$ if $P(\mathfrak{U}) \cap \mathscr{U} \neq\{0\}$. But, then $P(Q)=Q$ and so $Q \leq P$. However, $P$ is a minimal projection of $L(\mathscr{U})$ and so $Q=P$, a contradiction. Hence, $P(\mathscr{U}) \quad \mathscr{U}=\{0\}$. Virtually the same example is given on p. 59 of [2], a lthough the above example was discovered independently.

1.5 Proposition. Let $\mathcal{Q}$ be a generalized Hilbert algebra such that $\pi(\mathfrak{U})$ is a left ideal in $L(\mathscr{U})$. Then $\mathscr{U}$ is strongly orthocomplemented.

Proof. Let $\mathcal{T}$ be a closed right ideal of $\mathcal{U}$. Then $\mathcal{H}=\overline{\mathfrak{T}}^{\boldsymbol{H}} \oplus \mathcal{T}^{\Phi}$. Let $\xi \in \mathscr{U}$ with $\xi=\zeta+\Re_{\text {where }} \zeta \in \mathfrak{T}^{\mathcal{H}}$ and $\Re \in \mathcal{T}^{\oplus}$. Let $P$ be the orthogonal projection on $\mathcal{T}^{\mathcal{H}}$. Then as in the previous lemma $p \xi=\zeta \in \mathfrak{A} \cap \overline{\mathfrak{T}}^{\mathcal{H}}=\mathcal{T}$. Therefore, $\Re \in \mathscr{U} \cap \mathcal{T}^{\Theta}=\mathfrak{I}^{\perp}$ and so $\mathfrak{U}=\mathfrak{T} \oplus \mathfrak{I}^{\perp}$.

Let $P$ be a nonzero projection in $L(Q)$. Since $P(\mathscr{U}) \subseteq \mathscr{U}$ by hypothesis and $P(\mathfrak{U}) \neq\{0\}, P(\mathfrak{U}) \cap \mathfrak{U} \neq\{0\}$.

The previous arguments are only slight modifications of those in [7]. Thus, it is perhaps surprising that Proposition 1.5 has a converse.

1.6 Lemma. Let if be a generalized Hilbert algebra which is strongly orthocomplemented. If $P$ is any projection in $L(\mathfrak{Q})$ then $P(\mathscr{Q}) \subseteq \mathfrak{U}$.

Proof. It is first shown that $P(\mathscr{R}) \cap \mathscr{U}$ is dense in the range of $P$. Let $\mathcal{K}=$

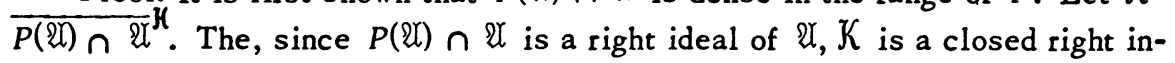
variant subspace of $\mathcal{H}(\mathscr{U})$ by Lemma 1.1. If $Q$ is the projection on $\mathcal{K}$ then $Q \epsilon$ $L(\mathscr{U})$ and $Q \leq P$. Let $P-Q=R$ a projection in $L(\mathscr{U})$. If $R \neq 0$ then there is a nonzero element $\xi \in \mathbb{U}$ such that $R \xi=\xi$. But, then $P \xi=\xi$ since $P \geq R$ and so $\xi \in \mathcal{K}$. Thus, $\xi=Q \xi=P \xi-R \xi=0$, a contradiction. Hence, $P(\mathscr{V}) \cap \mathscr{V}$ is dense in the range of $P$.

Let $\mathcal{T}=P(\mathscr{U}) \cap$ $U$. Since $\mathcal{T}$ is a closed right ideal of $\mathscr{U}$ one has $\mathscr{U}=\mathfrak{T} \oplus$ $\mathfrak{T}^{\perp}$. But since $\mathcal{T}$ is dense in the range of $P, \mathcal{T}^{\perp}$ is contained in the orthogonal complement of the range of $P$. Hence, $P(\mathscr{U})=P(\mathfrak{T})=\mathfrak{T} \subseteq \mathfrak{X}$.

1.7 Theorem. Let थ be a generalized Hilbert algebra which is strongly orthocomplemented. Then $\mathfrak{U}=\mathfrak{U}_{1} \oplus^{\perp} \mathfrak{U}_{2}$ where $\mathfrak{U}_{1}$ is a commutative strongly ortho. complemented Hilbert algebra and $\mathfrak{U}_{2}$ is a (strongly ortbocomplemented) generalized Hilbert algebra such that $\pi\left(\mathscr{U}_{2}\right)$ is a left ideal in $L\left(\mathscr{U}_{2}\right)$. 
Proof. Let $P$ be the largest abelian central projection in $L(\mathscr{U})$. Since $\mathscr{U}$ is strongly orthocomplemented, it is easy to see that $\mathscr{U}_{1}=P(\mathscr{U})$ and $\mathscr{U}_{2}=(1-P)(\mathscr{U})$ are closed two-sided ideals of $\mathfrak{U}$ and $\mathfrak{U}=\mathfrak{I}_{1} \oplus^{\perp} \mathfrak{U}_{2}$. It is not difficult to see that each of $\mathscr{U}_{1}$ and $\mathscr{U}_{2}$ is invariant under \# and hence they are generalized Hilbert algebras. Clearly, $\mathfrak{U}_{1}$ and $\mathscr{U}_{2}$ are strongly orthocomplemented and $\mathscr{U}_{1}$ is commutative. To see that $\mathfrak{U}_{1}$ is a Hilbert algebra, let $\left\{\xi_{\alpha}\right\}_{a \in A}$ be a left approximate identity in $\mathscr{U}_{1}$ and let $\pi, \zeta \in \mathscr{U}_{1}$. Then,

$$
\begin{aligned}
(\Re \mid \zeta) & =\lim _{a}\left(\xi_{a} \pi \mid \zeta\right)=\lim _{a}\left(\Re \xi_{a} \mid \zeta\right)=\lim _{a}\left(\xi_{a} \mid \Re^{\#} \zeta\right)=\lim _{a}\left(\xi_{a} \mid \zeta \pi^{\#}\right) \\
& =\lim _{a}\left(\zeta^{\#} \xi_{a} \mid \pi^{\#}\right)=\lim _{a}\left(\xi_{a} \zeta^{\#} \mid r^{\#}\right)=\left(\zeta^{\#} \mid r^{\#}\right) .
\end{aligned}
$$

Hence $\mathscr{U}_{1}$ is actually a Hilbert algebra. Now, let $Q \in L\left(\mathscr{U}_{2}\right)$ be a projection. Then by Lemma 1.6, $Q\left(\mathscr{U}_{2}\right) \subseteq \mathfrak{U}_{2}$. Now, one can identify $L\left(\mathscr{U}_{2}\right)$ and $(1-P) L(\mathscr{U})$ so that $L\left(\mathscr{R}_{2}\right)$ has no nonzero abelian direct summands. Therefore, by a theorem of Fillmore and Topping [3], $L\left(\mathscr{Q}_{2}\right)$ is generated algebraically by its projections. That is, $T\left(\mathscr{U}_{2}\right) \subseteq \mathfrak{U}_{2}$ for all $T \in L\left(\mathscr{U}_{2}\right)$ which implies that $\pi_{2}\left(\mathscr{U}_{2}\right)$ is a left ideal in $L\left(\mathrm{P}_{2}\right)$.

1.8 Corollary. Let $\mathfrak{U}$ be a generalized Hilbert algebra such that $L(\mathfrak{U})$ bas no infinite-dimensional abelian direct summands. Then $2 \mathrm{U}$ is strongly orthocomple. mented if and only if $\pi(\mathfrak{U})$ is a left ideal in $L(\mathfrak{Q})$.

1.9 Example. A commutative strongly orthocomplemented Hilbert algebra \& such that $\pi(\mathscr{U})$ is not a left ideal in $L(\mathscr{U})$.

Let $\mathscr{U}$ be the algebra of all Lebesgue measurable functions on $[0,1]$ which assume only finitely many values. Give $\mathcal{Q}$ the usual pointwise operations and inner product. Then $\mathfrak{U}$ is strongly orthocomplemented but $\pi(\mathfrak{U})$ is not an ideal in $L(2)=\pi\left(L^{\infty}[0,1]\right)$.

1.10 Theorem. Let if be an achieved generalized Hilbert algebra. Then the following are equivalent:

1. $\mathfrak{U}=\mathrm{H}_{l}$.

2. $\pi(\mathfrak{U})$ is a left ideal in $L(\mathfrak{U})$.

3. $\mathcal{U}$ is strongly orthocomplemented.

Proof. Condition 1 implies condition 2 since $\pi\left(\mathcal{H}_{l}\right)$ is a left ideal in $L(\mathfrak{U})$ by Lemma 2.3 of [2]. Condition 2 implies condition 3 is just Proposition 1.5.

To see that condition 2 implies condition 1 , let $\xi \in \mathcal{H}_{l}$ and let $\pi(\xi)=U T$ be the polar decomposition of $\pi(\xi)$. Now $U$ is in $L(\mathscr{U})$ and so $T=U^{*} \pi(\xi)=\pi\left(U^{*} \xi\right)$ is in $\pi\left(\mathcal{H}_{l}\right)$. But, since $T$ is selfadjoint, $T \in \pi(\mathscr{U})$. That is, $T=\pi(\zeta)$ for some $\zeta \in \mathfrak{A}$. Thus, $\pi(\xi)=U \pi(\zeta)$ is in $\pi(\mathscr{U})$ by hypothesis and therefore $\xi \in \mathfrak{U}$. That is, $\mathfrak{U}=\mathfrak{H}_{l}$. 
To see that condition 3 implies condition 2 let $\mathfrak{U}$ be strongly orthocomplemented and let $\mathfrak{U}=\mathfrak{U}_{1} \oplus^{\perp} \mathfrak{U}_{2}$ where $\mathfrak{U}_{1}$ is a commutative Hilbert algebra and $\pi\left(\mathscr{U}_{2}\right)$ is a left ideal in $L\left(\mathscr{U}_{2}\right)$ by Theorem 1.7. By Lemma 1 of [5], $\mathscr{U}_{1}$ is a full Hilbert algebra and so $\pi\left(\mathscr{U}_{1}\right)$ is an ideal in $L\left(\mathscr{U}_{1}\right)$. Hence, $\pi(\mathscr{U})$ is an ideal in $L(\mathscr{U})=L\left(\mathscr{U}_{1}\right) \oplus L\left(\mathscr{U}_{2}\right)$.

Clearly, $\mathfrak{H}_{l}$ itself is always strongly orthocomplemented so that condition 1 is not too surprising. Next, a useful inequality is obtained which tells when an achieved algebra is strongly orthocomplemented.

1.11 Theorem. Let $\mathfrak{U}$ be an achieved generalized Hilbert algebra. Then $\mathfrak{U}$ is strongly ortbocomplemented if and only if there is a $K>0$ sucb that for all $\xi \in \mathcal{U},\left\|\xi^{\sharp}\right\| \leq K(\|\xi\|+\|\pi(\xi)\|)$.

Proof. Suppose that $\mathscr{U}$ is strongly orthocomplemented, then by Theorem 1.10, $\mathfrak{U}=\mathcal{H}_{l}$. However, exactly as in Proposition 1.15 of [4], it is seen that $\xi \mapsto\|\xi\|+$ $\left\|\xi^{\sharp}\right\|+\|\pi(\xi)\|$ defines a Banach algebra norm on $\mathcal{U}$ and $\xi \mapsto\|\xi\|+\|\pi(\xi)\|$ defines a Banach algebra norm on $\mathcal{H}_{l}$. Since $\mathcal{U}=\mathcal{H}_{l}$, the open mapping theorem shows that there is a constant $M>1$ such that for all $\xi$ in $2\|\xi\|+\left\|\xi^{\#}\right\|+\|m(\xi)\| \leq$ $M(\|\xi\|+\|\pi(\xi)\|)$. Therefore, $\left\|\xi^{\#}\right\| \leq(M-1)(\|\xi\|+\|\pi(\xi)\|)$ for all $\xi$ in 2 .

On the other hand, suppose that the condition holds. Let $\zeta \in \mathcal{H}_{l}$ and let $\pi(\zeta)=U \pi(\xi)$ be the polar decomposition of $\pi(\zeta)$. Now, as seen in the proof of Theorem 1.10, $\xi \in \mathfrak{A}$ and $\xi=\xi^{\#}$. For each positive integer $n$ let $f_{n}$ be defined by

$$
\begin{aligned}
f_{n}(t) & =1 / t, \quad t \geq 1 / n, \\
& =0, \quad t<1 / n .
\end{aligned}
$$

Then, $f_{n}(\pi(\xi)) \in L(\mathscr{U})$ and so $f_{n}(\pi(\xi)) \xi \in \mathcal{H}_{l}$. But, $\pi\left(f_{n}(\pi(\xi)) \xi\right)=f_{n}(\pi(\xi)) \pi(\xi)$ is a selfadjoint idempotent in $L(\mathcal{U})$ and so $e_{n}=f_{n}(\pi(\xi)) \xi \in \mathfrak{U}$. Since $\pi(\xi)$ is positive, $\left\{\pi\left(e_{n}\right)\right\}$ converges to the range projection of $\pi(\xi)$ in the strong operator topology. However, since $\mathfrak{O}^{\prime}$ contains an approximate identity, $\xi$ is the closure of the range of $\pi(\xi)$ and so $\lim _{n} e_{n} \xi=\xi$. But, $e_{n}$ and $\xi$ commute so that $\lim _{n} \xi e_{n}=\xi$.

Now, let $\epsilon$ be any positive number and let $e_{n}$ be such that $\left\|\xi e_{n}-\xi\right\|<\epsilon / 2$. Then, since $\zeta=U \xi,\left\|\zeta e_{n}-\zeta\right\|=\left\|U \pi(\xi) e_{n}-U \xi\right\| \leq\left\|\xi e_{n}-\xi\right\|<\epsilon / 2$. By the

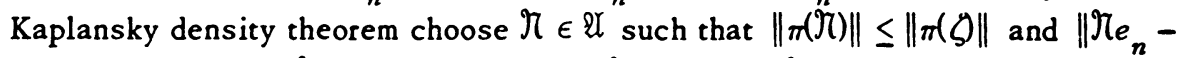
$\zeta e_{n} \|<\epsilon / 2$. Then, $\left\|\gamma e_{n}-\zeta\right\|<\epsilon$ and $\left\|\pi\left(\Re e_{n}\right)\right\| \leq\|\pi(\gamma)\| \leq\|\pi(\zeta)\|$. The above argument implies that there is a sequence $\left\{\zeta_{n}\right\} \subseteq \mathcal{Q}$ such that $\lim _{n} \zeta_{n}=\zeta$ and $\left\|\pi\left(\zeta_{n}\right)\right\| \leq\|\pi(\zeta)\|$. By assumption this implies that $\left\{\zeta_{n}^{\#}\right\}$ is a bounded sequence and hence by Alaoglu's theorem there is a subsequence $\left\{\zeta_{n_{k}}^{\#}\right\}$ and an element $\mu$ in $\mathcal{H}(\mathscr{U})$ such that $\zeta_{n_{k}}^{\#}$ converges to $\mu$ in $w^{*}$-topology of $\mathcal{H}(\mathscr{U})$. Thus, for all $\Re$ in $\mathfrak{U}^{\prime}$, 


$$
(\mu \mid \gamma)=\lim _{k}\left(\zeta_{n_{k}}^{\#} \mid \gamma\right)=\lim _{k}\left(\pi^{b} \mid \zeta_{n_{k}}\right)=\left(\pi^{b} \mid \zeta\right)
$$

Therefore, $\zeta$ is in the domain of \# and $\zeta^{\#}=\mu$. Thus, $\zeta \in \mathfrak{A}$ and so $\mathfrak{U}=\mathcal{H}_{l}$ which by Theorem 1.10 implies that $\mathcal{U}$ is strongly orthocomplemented.

1.12 Remark. If $\mathscr{U}$ is achieved and the involution is continuous on $\mathscr{U}$, then Ut is strongly orthocomplemented by the previous theorem (there are easier ways to see this). If the involution is not necessarily continuous but $\mathcal{A}$ has an identity 1 , then it is easy to see that for every $T$ in $L(\mathscr{U}), \pi(T(1))=T$ so that $\pi\left(\mathcal{H}_{l}\right)=$ $L(\mathfrak{U})$. But, then $\pi(\mathfrak{U})=\pi\left(\mathcal{H}_{l}\right) \cap \pi\left(\mathcal{H}_{l}\right) *=L(\mathfrak{U})$ so that $\mathfrak{X}=\mathcal{H}_{l}$ and therefore $\mathscr{U}$ is strongly orthocomplemented by Theorem 1.10 .

1.13 Tensor products. Let $\mathcal{H}$ and $\mathcal{K}$ be pre-Hilbert spaces and let $\mathcal{H} \otimes \mathcal{K}$ be the algebraic tensor product (over the complex numbers) of $\mathcal{H}$ and $\mathcal{K}$. Then $\mathcal{H} \otimes \mathcal{K}$ has a pre-Hilbert space structure given by

$$
\left(\sum_{k=1}^{n} x_{k} \otimes y_{k} \mid \sum_{j=1}^{m} w_{j} \otimes z_{j}\right)=\sum_{k=1}^{n} \sum_{i=1}^{m}\left(x_{k} \mid w_{j}\right)\left(y_{k} \mid z_{j}\right) .
$$

Now, if $\mathcal{U}$ and $\mathcal{B}$ are generalized Hilbert algebras then $\mathscr{U} \otimes \mathfrak{B}$ has a natural algebraic structure which makes $\mathcal{U} \otimes \mathcal{B}$ into an algebra with involution. To see that $\mathscr{U} \otimes \mathfrak{B}$ is a generalized Hilbert algebra with the above pre-Hilbert space structure, it suffices to see that the involution on $\mathscr{U} \otimes B$ is closable as a conjugate linear operator on $\mathcal{H}(\mathscr{U} \otimes \mathfrak{B})$. However, it is easy to see that $\mathcal{H}(\mathscr{U} \otimes \mathfrak{B})$ is just the completion of $\mathcal{H}(\mathscr{U}) \otimes \mathcal{H}(\mathscr{B})$ and so $\mathfrak{U}^{\prime} \otimes \mathfrak{B}^{\prime}$ is dense in $\mathcal{H}(\mathscr{U} \otimes \Re)$. Now, b (on $\mathfrak{U}^{\prime} \otimes \mathfrak{B}^{\prime}$ ) is a conjugate linear operator with domain dense in $\mathcal{H}(\mathscr{H} \otimes \mathfrak{B})$ and so has an adjoint. Clearly, the adjoint of $b$ extends the involution on $\mathscr{U} \otimes \mathfrak{B}$ and since the adjoint of a densely defined conjugate linear operator is closed, the involution on $\mathfrak{\imath} \otimes \mathfrak{B}$ is closable.

The following question can now be posed: Suppose $\mathscr{U}$ and $\mathfrak{B}$ are achieved generalized Hilbert algebras which are strongly orthocomplemented, is it then true that $(\mathscr{U} \otimes \mathfrak{B}) "$ is strongly orthocomplemented? Clearly, if both $\mathscr{V}$ and $\mathfrak{B}$ have an identity (both have a continuous involution) then $(\mathscr{U} \otimes \Re)$ " will have an identity (continuous involution) so that by the previous remark $(\mathscr{2} \otimes \Re)$ " will be strongly orthocomplemented. Moreover, Proposition 1.13 will show that $(\mathscr{C} \otimes \mathfrak{B})^{\prime \prime}$ can fail to be strongly orthocomplemented even if one chooses $\mathscr{U}$ to have an identity and $\mathfrak{B}$ to be an $H^{*}$ algebra. First, the following theorem (due essentially to Ambrose [1]) is needed. The proof here follows that of Rieffel [4].

1.14 Theorem. Let 2 be a generalized Hilbert algebra sucb that either OU is acbieved or $\pi(\mathfrak{Q})$ is a left ideal in $L(\mathfrak{Q})$. Then any nonzero left or right ideal of $\mathrm{U}$ contains a nonzero selfadjoint idempotent. 
Proof. The proof is given for right ideals only. Let $\mathcal{T}$ be a right ideal of $\mathscr{\imath}$ such that there is some $\xi \in \mathcal{T}$ with $\xi \neq 0$. Then, $\xi \xi^{\#} \in \mathcal{T}$ and $\xi \xi^{\#} \neq 0$. Let $\zeta=$ $\xi \xi^{\#}$; then $\pi(\zeta)$ is a positive selfadjoint operator in $L(2)$. Let $J$ be a closed interval of the real line whose interior meets the spectrum of $\pi(\zeta)$ but such that $0 \notin J$. Define

$$
\begin{aligned}
f(t) & =1 / t^{2}, \quad \text { for } t \in J, \\
& =0, \quad \text { for } t \notin J .
\end{aligned}
$$

Then, $T=f(\pi(\zeta)) \in L(\mathscr{U})$. Now, if $\mathcal{U}$ is achieved then since $T \zeta \in \mathcal{H}$, and $\pi(T \zeta)=$ $f(\pi(\zeta))_{\pi}(\zeta)$ is a selfadjoint operator, $T \zeta \in \mathscr{U}$. On the other hand if $\pi(\mathscr{U})$ is a left ideal $L(\mathscr{U})$ then, clearly, $T \zeta \in \mathscr{U}$. Therefore, $\zeta T \zeta \in \mathcal{T}$, but, $\pi(\zeta T \zeta)=f(\pi(\zeta)) \pi(\zeta)^{2}$ is a nonzero selfadjoint projection in $L(\mathscr{U})$ and so $\zeta T \zeta$ is a nonzero selfadjoint idempotent in $\mathfrak{T}$.

1.15 Proposition. Let $\mathcal{A}$ and $\mathfrak{B}$ be achieved generalized Hilbert algebras which are strongly orthocomplemented. Suppose that $\mathcal{O}$ bas a discontinuous involution and that $\mathfrak{B}$ does not have an identity. Then $(\mathscr{Q} \otimes \Re)$ " is not strongly ortbocomplemented.

Proof. We first show that the set of projections in $\mathcal{B}$ is unbounded. Let $\mathcal{E}$ be a maximal family of orthogonal projections in $B$. Then, since $\mathscr{B}$ is strongly orthocomplemented one can use the previous theorem and the maximality of $\mathcal{E}$ to show that $B=\overline{\Sigma_{e \in E^{e}}}$. Now, if the set of projections in $B$ were bounded, say, $K>0$ then for every finite subset $\mathcal{F}$ of $\mathcal{E}$,

$$
\sum_{e \in \mathcal{F}}\|e\|^{2}=\left\|\sum_{e \in \mathcal{F}} e\right\|^{2} \leq K^{2} .
$$

That is, $\Sigma_{e \epsilon \in \mathbb{G}}\|\|^{2}$ would converge and so $\Sigma_{e \in \varepsilon^{e}}$ would define a selfadjoint pro-

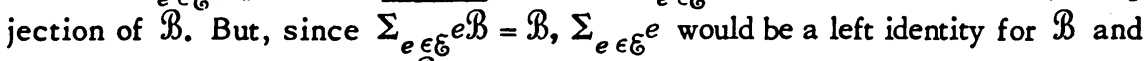
therefore an identity. Since $\mathfrak{B}$ has no identity by hypothesis, the set of projections in $\mathbb{B}$ is unbounded.

Now, since the involution on $\mathcal{U}$ is discontinuous, let $\left\{\xi_{n}\right\} \subseteq \mathcal{Q}$ be a sequence such that $\lim _{n} \xi_{n}=0$ but $\left\|\xi_{n}^{\#}\right\|=1$ for all $n$. Now, choose a sequence of selfadjoint idempotents $\left\{e_{n}\right\} \subseteq \mathfrak{B}^{n}$ such that $\left\|e_{n}\right\|>n\left\|\pi\left(\xi_{n}\right)\right\|$. If $(\mathscr{Q} \otimes \mathfrak{B})$ " were strongly orthocomplemented then by Theorem 1.11 there would exist a constant $K>0$ such that $\left\|\zeta^{\sharp}\right\| \leq K(\|\zeta\|+\|\pi(\zeta)\|)$ for all $\zeta$ in (थ $\left.\otimes \Re\right)^{\prime \prime}$. But, then $\left\|\xi_{n}^{\#} \otimes e_{n}\right\|$ $\leq K\left(\left\|\xi_{n} \otimes e_{n}\right\|+\left\|\pi\left(\xi_{n} \otimes e_{n}\right)\right\|\right)$ or $0<1 / K \leq\left\|\xi_{n}\right\|+\left\|\pi\left(\xi_{n}\right)\right\| /\left\|e_{n}\right\|<\left\|\xi_{n}\right\|+1 / n$. for all positive integers $n$ which is a contradiction. Hence, $(\mathscr{Z} \otimes \mathscr{B})^{n}$ is not strongly orthocomplemented.

1.16 Direct sums. Let $\left\{\mathscr{U}_{a}\right\}_{a \in A}$ be a collection of generalized Hilbert algebras. Then the algebraic direct sum of $\left\{\mathscr{U}_{a}\right\}_{a \in A}$ is clearly a generalized Hilbert 
algebra with the obvious operations and inner product. This algebra is denoted by $\Sigma_{a \in A} \mathfrak{T}_{a}$. Clearly $\Sigma_{a \in A} \mathfrak{H}_{a}$ is strongly orthocomplemented if and only if each $\mathfrak{U}_{a}$ is strongly orthocomplemented. However, each $\mathfrak{U}_{a}$ can be achieved and strongly orthocomplemented while $\left(\Sigma_{a \in A} \mathfrak{R}_{a}\right)$ " is not strongly orthocomplemented, as seen below.

1.17 Example. Let $\mathcal{H}$ be a separable Hilbert space with orthonormal basis $\left\{x_{n}\right\}_{n=1}^{\infty}$. Let $\mathfrak{U}$ be the set of all bounded linear operators on $\mathcal{H}$ and let \# denote the usual operator adjoint. Give $\mathscr{U}$ the following inner product:

$$
(\xi \mid \eta)=\sum_{k=1}^{\infty} \frac{1}{2^{k}}\left(\xi x_{k} \mid \pi x_{k}\right) \text { for } \xi, \pi \in \Re \text {. }
$$

Then, $\mathscr{A}$ is an achieved generalized Hilbert algebra with identity and therefore is strongly orthocomplemented. Let $B=\left(\sum_{n=1}^{\infty} \mathfrak{l}\right)$ ". For each positive integer $n$, let $\xi_{n} \in \mathscr{U}$ be defined by $\xi_{n}(z)=\left(z \mid x_{n}\right) x_{1}$ for all $z \in \mathcal{H}$. Then, for each $n,\left\|\xi_{n}\right\|^{2}$ $=1 / 2^{n}$, but $\left\|\xi_{n}^{\#}\right\|^{2}=1 / 2$ so that $\sum_{n=1}^{\infty}\left\|\xi_{n}\right\|^{2}<\infty$, but $\sum_{n=1}^{\infty}\left\|\xi_{n}^{\#}\right\|^{2}=\infty$. Moreover $\left\|\pi\left(\xi_{n}\right)\right\| \leq 1$ for each $n$ so that is it easy to see that $\left\{\xi_{n}\right\}_{n=1}^{\infty} \in \mathcal{H}_{l}$ but is not in $\mathfrak{B}$. By Theorem $1.10, \mathcal{B}$ is not strongly orthocomplemented. One notes that $\mathcal{B}$ satisfies the second but not the first condition in the definition of strongly orthocomplemented. If one takes the direct sum of $\mathfrak{B}$ with the example in 1.4 , one obtains an achieved generalized Hilbert algebra which satisfies neither condition of the definition.

1.18 The generalized Hilbert algebra of a group. Let $G$ be a locally compact group with left Haar measure $\mu$ and modular function $\Delta$. Let $\mathfrak{U}_{0}(G)$ be the (convolution) algebra of all continuous complex valued functions on $G$ with compact support. Equip $\mathfrak{U}_{0}(G)$ with the following inner product and involution:

$$
\begin{aligned}
& (f \mid g)=\int_{G} f(x) \overline{g(x)} d \mu(x), \\
& f^{\#}(y)=\Delta\left(y^{-1}\right) \overline{f\left(y^{-1}\right)} \text { for all } y \in G, \text { and for all } f, g \in \mathscr{U}_{0}(G) .
\end{aligned}
$$

Then, $\mathfrak{U}_{0}(G)$ is a generalized Hilbert algebra. Define $\mathfrak{\mathscr { U }}(G)=\left(\mathscr{U}_{0}(G)\right)$ " and call $\mathscr{U}(G)$ the generalized Hilbert algebra of $G$.

If $G$ is unimodular, then $\mathscr{U}(G)$ is a full Hilbert algebra and so is strongly orthocomplemented. On the other hand, if $G$ is not unimodular then $\mathscr{U}(G)$ is not strongly orthocomplemented. To see this, let $G$ be unimodular so that $\Delta$ is unbounded on $G$. Since $G$ is not discrete, for each positive integer $n$ there is an open set $W_{n} \subseteq G$ with compact closure such that $\mu\left(W_{n}\right) \leq 1$ and $\Delta(x)>n^{2}$ for all $x \in W_{n}$. Now, the characteristic function $\chi_{W_{n}}$ of $W_{n}$ is clearly in the domain of \# and the operator $g \mapsto \chi_{W_{n}} * g$ is bounded for $g \in L^{2}(G)=\mathcal{H}(\mathcal{2}(G))$ by $\mu\left(W_{n}\right) \leq 1$. Thus, $\chi_{W_{n}} \in \mathcal{U}(G)$ for each postive integer $n$. Now, an easy calculation shows that 


$$
=\int_{G} \Delta(x) \chi_{W_{n}}(x) d \mu(x) \geq n^{2} \mu\left(W_{n}\right) .
$$

If $\mathcal{U}(G)$ were strongly orthocomplemented, then by Theorem 1.11 there would exist $K>0$ such that for each $n$

$$
n \mu\left(W_{n}\right)^{1 / 2} \leq\left\|\chi_{W_{n}}^{\#}\right\| \leq K\left(\left\|\chi_{W_{n}}\right\|+\left\|\pi\left(\chi_{W_{n}}\right)\right\|\right) \leq K\left(\mu\left(W_{n}\right)^{1 / 2}+\mu\left(W_{n}\right)\right) .
$$

Or, $n \leq K\left(1+\mu\left(W_{n}\right)^{1 / 2} \leq 2 K\right.$ for all positive integers $n$ which is a contradiction. Hence $\mathfrak{U}(G)$ is not strongly orthocomplemented.

I would like to thank J. P. Williams for pointing out the existence of Fillmore and Topping's paper [3].

\section{BIBLIOGRAPHY}

1. W. Ambrose, The $L^{2}$-system of a unimodular group. I, Trans. Amer. Math. Soc. 65 (1949), 27-48. MR 10, 429.

2. F. Combes, Poids associé à une algèbre Hilbertienne à gauche, Compositio Math. 23 (1971), 49-77. MR 44 \#5786.

3. P. A. Fillmore and D. M. Topping, Operator algebras generated by projections, Duke Math. J. 34 (1967), 333-336. MR 35 \#51.

4. M. A. Rieffel, Square-integrable representations of Hilbert algebras, J. Functional Analysis 3 (1969), 265-300. MR 39 \#6094.

5. M. Takesaki, Conditional expectations in von Neumann algebras, J. Functional Analysis 9 (1972), 306-321.

6. - Tomita's theory of modular Hilbert algebras and its applications, Lecture Notes in Math., vol. 128, Springer-Verlag, Berlin and New York, 1970. MR 42 \#5061.

7. B. Yood, Hilbert algebras as topological algebras, Ark. Mat. 12 (1974), 131-151.

SCHOOL OF MATHEMATICS, THE UNIVERSITY, NEWCASTLE UPON TYNE, ENGLAND

Current address: Department of Mathematics, Queen's University, Kingston, Ontario, Canada K7L 3N6 